\title{
Das Wissen und seine Gesellschaft
}

\author{
Michael Jäckel
}

\section{Zusammenfassung}

Als Diderot im 18. Jahrhundert sein Projekt einer Encyclopédie entwickelte, galt es als Versuch, den Kontinent zu erleuchten. Der Zugang zu Wissen sollte sich verändern - ein Beispiel aus vielen, das den Zusammenhang von Wissensordnung und Medienordnung zu verdeutlichen vermag. Dieser Prozess lässt sich historisch auch als eine Zunahme von Formenvielfalt beschreiben.

Eine Formenvielfalt muss Institutionen, die eine Wissensordnung repräsentieren, auch dazu veranlassen, herkömmliche Strukturen mit neuen Optionen zu vergleichen. Das vollzieht sich aktuell im Hochschulwesen. In Lehre und Forschung entwickeln sich dadurch potenziell neue Reputationsfelder, auch außerhalb der herkömmlichen „Räume“. Der Beitrag analysiert diese Übergangssituation und verdeutlicht, wie diese Impulse in existierende Wissensordnungen einfließen.

\section{Schlüsselwörter}

Aufklärung • Didaktik • Inklusion • Licht • Reputation • Selbstorganisation • Wahrheit $\bullet$ Wissen $\bullet$ Wissensgesellschaft

M. Jäckel ( $\varangle)$

Universität Trier, Trier, Deutschland

E-Mail: jaeckel@uni-trier.de 


\section{$1 \quad$ Wissen als Kategorie und Vorstellung}

Der Begriff „Wissen“ genießt den Status einer soziologischen Kategorie. Er steht für die Gewissheit von Erfahrungen, für eine Differenzierung je nach sozialem Standort und für eine soziale Ordnung, die dadurch bestimmt wird (vgl. Knoblauch und Tuma 2018, S. 517). Seine Prominenz lässt sich auch daran ablesen, dass er einem Gesellschaftstypus einen Namen verleiht. So wie wir die Erlebnis-, Risiko-, Leistungs-, Spaß- oder Protestgesellschaft kennen, reklamiert die Wissensgesellschaft für sich die besondere Wertschätzung dieses Phänomens als zentrale Ressource (vgl. ebenda, S. 519). Zugleich verleiht die Kategorie einer speziellen fachlichen Ausrichtung, der Wissenssoziologie, ihren Namen.

$\mathrm{Zu}$ der Vorstellung einer zentralen Ressource gehört unweigerlich die Frage, wie dieses Wissen entsteht und wie der Zugang dazu geregelt wird. Nicht weniger wichtig ist die Frage, welche Lebenschancen an dessen Verwertung gekoppelt sind. Wissen lenkt daher die Aufmerksamkeit immer auch auf die Struktur des Wissensbetriebs. Mit Blick auf die lange Geschichte der Universität ist beispielsweise die Resilienz dieser Institution als Organisationsform des Wissens immer wieder betont worden. Die Strukturen, die dieser Typus historisch entwickelt hat, sind stets notwendig im Sinne des Erhalts und der Wahrung des Auftrags interpretiert worden. Das Wachstum der Erkenntnis soll kontrolliert erfolgen, die Wissenschaft muss ihre Komplexität ordnen können und über anerkannte Reputationspfade Integration gewährleisten. Auf dieses wiederkehrende Element hat insbesondere Rexrodt (2019) hingewiesen. Die Diskussion ist im Zuge der Digitalisierung neu belebt worden. Aber jene Diskussion fiel nicht aus heiterem Himmel in eine darauf unvorbereitete Situation, sondern hat selbst eine Geschichte, die zumindest in Auszügen - und ohne Anspruch auf Vollständigkeit - hier erwähnt werden soll.

Vor 50 Jahren sprach kaum jemand von Digitalisierung, aber vom Aufkommen der Informationsgesellschaft. Daniel Bell war einer der bekanntesten Repräsentanten und gelangte mit seinem Buch über die postindustrielle Gesellschaft zu hoher Popularität. Dem Buch voraus ging die Einrichtung einer „Kommission für das Jahr 2000“, eingerichtet von der American Academy of Arts and Sciences (vgl. hierzu ausführlicher Pias 2019, S. N4). Wissen erhielt hier den Status eines axialen Prinzips. Der Motor des gesellschaftlichen Wandels wird von dieser Achse repräsentiert, um die sich sozusagen alles dreht. Hätte Bell diese Beschreibung auf die digitale Gesellschaft angewandt, müsste ihm heute kaum widersprochen werden. Das Phänomen hat sich nicht nur bewegt, sondern einen deutlichen Sprung gemacht. 
Wenige Jahre nach der Veröffentlichung von Daniel Bells Grundlagenwerk erschien eine kompakte Abhandlung des französischen Philosophen Jean-François Lyotard, die aus einem Gutachten für die kanadische Regierung hervorging. $\mathrm{Zu}$ Beginn des Jahres 2020 wurde dieser Analyse eine fast visionäre Kraft für Fragen zugeschrieben, die aktuell diskutiert werden.

„Betrachtet man die Liste seiner Beobachtungen dazu, fühlt man sich in der Gegenwart. Die Informatik, schreibt er, sei auf dem Vormarsch und mit ihr Disziplinen wie Computerlinguistik, Nachrichtentechnik, Übersetzungs- und Automatentheorie. Fragen der Speicherung von Daten, der Aufwertung von Information und der,Perfektionierung intelligenter Terminals“ würden vordringlich“" (Kaube 2020, S. 33).

In der Tat wird der geordneten Struktur der Fachdisziplinen an vielen Stellen entgegengehalten, dass die „Enzyklopädie des deutschen Idealismus“ (Lyotard 1999 [zuerst 1982], S. 103) mit Datenbanken als den Enzyklopädien von morgen (vgl. ebenda, S. 151) konkurrieren werde; Verwirrungen im Curriculum seien das Ergebnis einer wachsenden Bedeutung von Interdisziplinarität. Nicht mehr die Professor*innen stehen im Mittelpunkt, sondern das Forschungsteam. Die Telematik wird zu einem Teil einer höheren Propädeutik erklärt (vgl. ebenda, S. 149), es gehe nicht mehr um die Vermittlung von Idealen, sondern um Kompetenzen. Auch der Satz „Der Student hat sich schon verändert und wird es noch müssen“ (ebenda, S. 142) steht für eine neue Performanz der Universität, die sich von dem Anspruch lösen müsse, für die Emanzipation im Sinne des sozialen Fortschritts auszubilden, und auf berufsqualifizierende Funktionen umstellen (vgl. ebenda, S. 142 ff.). Vernetzung, Steuerung, Interdependenz - die Semantik dieses Wandels stellt das klassische akademische Subjekt unter Druck. In den 1960er-Jahren klang das bei McLuhan so: „Die Souveränität mancher Lehrkanzel ist unter den von der elektrischen Geschwindigkeit geschaffenen Bedingungen so rasch geschwunden wie die manch eines Nationalstaats" (McLuhan 1968, S. 44 f.). Eher unbeeindruckt von diesem massiven Einschnitt in die Personengebundenheit des Denkens betonte noch Robert King Merton im Jahr 1972 ein Normen- und Wertesystem der Wissenschaft, das von folgenden Prinzipien getragen werde:

- Universalismus: Der Geltungsanspruch von Wissenschaft kennt keine Verbindungen zu persönlichen Vorlieben oder beeinflussende Effekte sozialer Kategorien wie beispielsweise Religion oder Klasse.

- Gemeinschaftlichkeit: Die Ergebnisse wissenschaftlichen Arbeitens stehen der Gemeinschaft zur Verfügung und unterliegen einem treuhänderischen Auftrag. Jede akademische Kohorte trägt zu diesem Erbe bei. 
- Uneigennützigkeit: Die Regeln wissenschaftlichen Arbeitens schützen vor einer Vorteilsnahme auf der Basis unerlaubter Mittel und gewährleisten Öffentlichkeit und Überprüfbarkeit.

- Organisierter Skeptizismus: Wissenschaft legt Wert auf Reliabilität, also auf die Möglichkeit der Überprüfung von „Tatsachenbehauptungen“ unter Einbindung etablierter Verfahren (Prüfverfahren, Gutachterwesen) (vgl. Merton 1972 sowie Knoblauch 2008, S. 476).

Daraus folgt: Wer das Wissen und seine Gesellschaft beobachten möchte, der muss in der Lage sein, Grenzen zu ziehen. Was im Jahr 2020 in unendlich vielen Köpfen an Ideen, Vorstellungen, geprüften und ungeprüften Lösungen für vorhandene und noch nicht vorhandene Probleme existiert, deckt sich natürlich nicht mit dem, was wir als einen „Kanon des Wissens“ bezeichnen würden. Aber Versuche, dieses Wissen zu bündeln, unterlagen in der Vergangenheit den Kontroll- und Hegemonieansprüchen starker, meist kirchlicher Institutionen. Bevor der gerade beschriebene Wertekanon sich entfalten konnte, musste vieles zunächst ans Licht gebracht werden.

\section{Licht, Wissen und Wahrheit}

In Hans Blumenbergs Essay „Licht als Metapher der Wahrheit“ werden die Geschichte einer Verwandtschaft und die Geschichte einer viel verwandten Metapher anschaulich beschrieben und analysiert. Die Bedeutung des Lichts hat Eingang in die Formulierung verschiedenster Gleichnisse erhalten, wovon das Höhlengleichnis Platons eines der bekanntesten sein dürfte. Menschen nehmen darin nur die Schatten von bestimmten Phänomenen wahr und verbleiben, solange sie nicht ans Licht geführt werden, in einem Zustand mangelnder Erkenntnis. In der Metapher des Lichts fließt gleichsam eine Vorstellung von und Sehnsucht nach Wahrheit zusammen. In den Worten Blumenbergs:

„Licht kann der gerichtete Strahl, die wegweisende Leuchte im Dunkel, die vordringende Entmachtung der Finsternis, aber auch die blendende Überfülle, ebenso wie die unbestimmbar allgegenwärtige Helle sein, in der alles darinsteht: das selbst nichterscheinende Erscheinenlassen, die unzugängliche Zugänglichkeit der Dinge. Licht und Finsternis können die absoluten metaphysischen Gegenmächte repräsentieren, die sich ausschließen und doch das Weltgefüge zustande bringen“ (Blumenberg 2001, S. 140). 
Das Spiel mit dem Licht verdeutlicht zugleich, dass man der Vorstellung, Wissen könnte etwas Abgeschlossenes, etwas Hermetisches, etwas, das Anfang und Ende kennt, sein, eine Absage erteilen muss. Das ist auch der große Gedanke der Aufklärung, dessen „Abstammung aus der Lichtsprache“ (ebenda, S. 168) im Deutschen gleich mehrfach ersichtlich ist. Der französische Begriff verdeutlicht es ebenso: „siècle des lumières“. Im Englischen steht dafür der Begriff „enlightenment“ (vgl. ebenda, S. 168).

Dennoch ist, wenn es um den Zusammenhang von Wissen und Gesellschaft geht, alleine die Bestimmung des Anfangs einer Debatte ausgesprochen schwierig. Wenn im Zusammenhang mit einer Öffnung der Welt des Wissens in jüngerer Zeit auch der Begriff „digitaler Humanismus“ in Verwendung kam, dann wird unsere Aufmerksamkeit alleine durch den Namen auf die Übergangsphase des Spätmittelalters in die Frühe Neuzeit gelenkt. Als im Jahre 2019 zahlreiche Beiträge das Wirken Leonardo da Vincis würdigten, galt diesem Wunsch nach Öffnung und seiner Artikulation insbesondere im 15. Jahrhundert die Aufmerksamkeit. „Denken ist keine Sünde" lautete ein Beitrag von Assheuer, in dem die Bestrebungen, die Kontrollmacht der Kirche über das Wissen der Welt zu reduzieren, in ein Plädoyer zur „freien Welterforschung“ mündeten (Assheuer 2019, S. 42). Dieser Erweiterungsgedanke ging einher mit der Vorstellung, die Entstehung des Wissens der Welt nicht zu monopolisieren, sondern viele an der Entschlüsselung unbekannter Dinge teilhaben zu lassen: ,,...] und weil im menschlichen Intellekt der göttliche Ursprung fortwirkt, könne es dem höchsten Künstler nicht gefallen, wenn die Herrlichkeit seiner Schöpfung unerkannt bleibe“ (ebenda, S. 41). Das war die Idee des Humanismus und sie musste gegenüber vielen Anfeindungen verteidigt werden.

Ein weiteres prominentes Beispiel ist das Bestreben, das Wissen der Welt in einer Enzyklopädie zusammenzufassen. Es ist mit dem Namen französischer Philosophen der zweiten Hälfte des 18. Jahrhunderts verbunden und gilt als eines der Großprojekte der Aufklärung. Zugleich zeigt eine nähere Betrachtung seiner Geschichte, dass zwischen diesem Ideal, nämlich Wissen als ein Referenzsystem für die Gesellschaft aufzubauen, und dem Zugang zu diesem Wissen sich eine nicht unerhebliche Kluft auftat. Auch die große Enzyklopädie von Diderot und d'Alembert war letztlich ein Prestigeprojekt, dessen Wirkung im Zuge der Französischen Revolution auch rasch verpuffte. Aber Wissen eroberte damit weitere Teile der Öffentlichkeit und verstärkte die Erosion geschützter Wissensräume.

Ein kurzer Blick auf die Institutionen, in denen Wissensvermittlung stattfand, verdeutlicht darüber hinaus Debatten über inhaltliche Schwerpunktsetzungen, aber auch über didaktische Konzepte. So lautete im Spätmittelalter ein häufiger Vorwurf „scholastische Erstarrung“, Ende des 18. Jahrhunderts war es die 
Langeweile des Kathedervortrags, die die Kritik am universitären Alltag symbolisierte. Die Idee von Akademien wurde als Alternative diskutiert, der neue Idealtypus der deutschen Universität musste im 19. Jahrhundert auf die wachsende funktionale Differenzierung, die sich auch in einer Spezialisierung der Disziplinen niederschlug, reagieren. Rückblickend werden die großen Reformen des 19. Jahrhunderts als wegweisende Veränderungen der bedeutenden Orte des Wissens interpretiert. Wenn zu Beginn des 21. Jahrhunderts hier und da der Ruf nach einem neuen Humboldt laut wird, findet die wahrgenommene Tragweite der Veränderungen Ausdruck in einer prominenten Forderung. Gerne wählt die Wissenschaft für radikale Perspektivenwechsel auch den englischen Begriff „turn“. Jedes Mal, wenn er auftaucht, werden weitreichende und nachhaltige Veränderungen beschrieben, die gleichsam das Grundgerüst wissenschaftlichen Arbeitens betreffen.

\section{Das Wissen und seine Vermittlung}

Die Ausführungen haben bis hierhin gezeigt, dass der Wandel von Institutionen des Wissens die Rollenverständnisse der Beteiligten berührt, den Zugang zu und die Lösung wissenschaftlicher Problemstellungen verändert, Grenzziehungen zwischen Disziplinen betrifft und eine Konkurrenz der Vermittlungsformen dieses Wissens entstehen lässt. Gegenwärtig dominiert noch ein Reichtum an interessanten Produkten. Eine Architektur fehlt noch, die diesen unkontrollierten Innovationsschub, der alljährlich auf IT-Gipfeln, Fachmessen usw. neue Impulse erhält, in einen abgestimmten Schaltplan überführt. Es soll ausdrücken, dass die Koexistenz alter und neuer Strukturen in eine neue Balance überführt werden muss. Diese neue Balance soll fortan im Mittelpunkt stehen. Sie wird entlang der folgenden Dimensionen diskutiert: Lehrende und Lernende, Reputationsstrukturen, Wertschätzung von Didaktik und Methoden/Forschungsdesigns.

\subsection{Lehrende und Lernende}

Regelmäßig wird daran erinnert, dass Lernen in hohem Maße außerhalb von Bildungseinrichtungen stattfindet. Die Vorstellung, Bildung finde vorwiegend innerhalb eines institutionellen und relativ klar strukturierten Rahmens statt, gilt nur für einen Teil dieses Lern- und Aneignungsprozesses. Der Tatbestand verweist auf die Notwendigkeit der Selbstorganisation des Lernens. Der Begriff „Selbstorganisation“ hat in den letzten Jahren eine erkennbare Renaissance erlebt, 
die insbesondere aus einer Neubestimmung des Verhältnisses von Menschen und Institutionen, aber auch aus einer verstärkten Betonung von Selbstverwirklichung und damit verbundenen Konsequenzen resultiert (vgl. hierzu Beck und Beck-Gernsheim 1994). Ebenso mag die hohe Selbstlernquote dazu einladen, den Prozess des Lernens in Institutionen hinsichtlich seiner Qualität zu hinterfragen. Heinz von Foerster, der als „Vater des Konstruktivismus“ für seine Analysen von Selbststeuerungs- und Selbstorganisationsprozessen bekannt ist, soll auf die Frage, was ihn an seiner Schulzeit besonders gestört habe, einmal geantwortet haben: diese Welt der Monologe. Wenn Dialoge nicht gestattet waren, so berichtet Reinhard Kahl in seinem Portrait „Der Neugierologe“, konnte er sehr bockig werden, zum Beispiel gegenüber seinem Mathematiklehrer. Als dieser ihn fragte: „Zwei Bauarbeiter brauchen zum Ausschachten einer Grube fünf Stunden. Wie viele Stunden brauchen drei Arbeiter?“ antwortete er: „Zehn Stunden, denn zu dritt gehen sie erst mal Karten spielen“ (zit. nach Kahl 1999, S. 107).

In einem weiteren Sinne könnte diese Kritik aber auch als Hinweis auf die Nichteinlösung eines aufklärerischen Ideals verstanden werden, das Reinhart Koselleck wie folgt beschrieb:

„Es kennzeichnet den deutschen Bildungsbegriff, daß er den Sinn einer von außen angetragenen Erziehung, der dem Begriff im 18. Jahrhundert noch innewohnt, umgießt in den Autonomieanspruch, die Welt sich selbst einzuverwandeln [...]“ (Koselleck 1990, S. 14).

Ideale dieser Art laden immer wieder dazu ein, Wunsch und Wirklichkeit zu kontrastieren.

Wer im universitären Alltag eine „Welt der Monologe“ dominieren sieht, sollte nicht ohne Widerspruch davonkommen. Wer eine Vorlesung vor Augen hat, die 90 min lang tatsächlich einem Vorlesen entspricht, mag zustimmen. Wer überfüllte Seminare kennt, in denen ein Referat nach dem anderen vorgetragen wird, ohne dass genügend Zeit für die Reflexion des Gehörten bleibt, wird ebenfalls mit dem Kopf nicken. Es gibt nach meinem Kenntnisstand keine objektive Messung über die Qualität des Hochschulunterrichts, die in dieser Hinsicht zu einem sachlichen Urteil beitragen könnte. Eher stehen sich Stereotype gegenüber. Wer einer bestimmten Situation erst einmal eine Bedeutung gegeben hat, neigt selten dazu, sein Folgeverhalten an neuen Wahrnehmungen oder veränderten Rahmenbedingungen auszurichten. Wer die universitäre Lehre aufmerksam verfolgt, erlebt das Aufkommen einer neuen Experimentierfreude, die nicht nur der Sorge um nachlassende Aufmerksamkeitsspannen zuzuschreiben ist, sondern einem wachsenden Arsenal an Gestaltungsmöglichkeiten unterschiedlichster Veranstaltungsformate. 
Damit einher geht eine Auflösung der Grenzen bestimmter Veranstaltungstypen. Eine Vorlesung kann Elemente eines Seminars übernehmen, Seminare können in Teilen Vorlesungscharakter haben, neue Formen der Einbindung des „Publikums“ gestatten Interaktionen vor, während und nach einer Veranstaltung. Experimente dieser Art können hier und da durchaus zu didaktischen Überdramatisierungen führen, aber aus einem Zuviel an Elementen lernen die Beteiligten relativ schnell, was weggelassen werden kann. Die digitale Lehre kann sich dabei sowohl auf eine Anreicherung der Präsenzlehre, auf die Gestaltung des Ablaufs einer Veranstaltung und inhaltlich durchaus auf neue Themen beziehen, die aufgrund der Digitalisierung möglich geworden sind. Der Möglichkeitsraum lässt sich entlang von vier Dimensionen beschreiben:

- In räumlicher Hinsicht wird Präsenz zu einem optionalen Kriterium. Die Vorlesung wird vorab aufgenommen, mit Zusatztools versehen und an verschiedenen Orten innerhalb und außerhalb der Universität rezipiert. Die eigentliche Präsenzveranstaltung fokussiert dann die Kommentare, Anmerkungen etc. der Studierenden zu dem bereits bekannten Vorlesungsinhalt. Das ist das Modell „Flipped Classroom“.

- Die Entkopplung von einem bestimmten Datum - die zeitliche Dimension mithin - meint die Ausweitung des Formats auf Zeiten vor, während und nach der Präsenzphase. Natürlich bleiben da zeitliche Restriktionen, aber Lernplattformen versetzen die Lehrenden und die Lernenden in die Lage, die Beschäftigung mit einem Thema während des Semesters auf Dauer zu stellen. Knappheitsprobleme treten auch hier auf, aber mit besonderem Engagement gelingen auch besondere Dinge.

- In sozialer Hinsicht verändert sich damit das Rollenverständnis der Lehrenden und der Lernenden. Die Moderatorenfunktion gewinnt an Bedeutung, denn ohne Beteiligung aufseiten der Studierenden verfehlt das gesamte Setting gleichwohl sein Ziel. Die Zwischenschaltung von Technik ist nicht immer notwendig, im Sinne einer Inklusion des Teilnehmerkreises aber von Vorteil.

- In sachlicher Hinsicht entsteht damit ein neuer Blick auf das Wissen der Gesellschaft. Der Mikrokosmos „Vorlesung“ oder „Seminar“ verknüpft Elemente des Monologs mit Elementen des Austauschs.

In kommunikationstheoretischer Hinsicht wird also bewusst auf „Noise“ gesetzt. Das Studium wird somit nicht durch den Ausschluss, sondern durch den Einschluss von Störungen in einer gewissen Weise weiterentwickelt.

Die Qualität des Angebots sowie die Vorkenntnisse und Fähigkeiten der Involvierten bestimmen den Erfolg. Synchrone und asynchrone Elemente ergänzen 
sich. Der Campus und die Universität setzen auf An- und Abwesenheit, die Infrastruktur lädt zu beidem ein.

\subsection{Wertschätzung der Didaktik}

Die Welt der Wissenschaft ist ein sehr wettbewerbsorientiertes System. Das Kriterium des Universalismus (siehe oben) betont in besonderer Weise den Verzicht auf Ungleichbehandlung. Anerkennung wird wissenschaftlicher Erkenntnis gezollt, die den Regeln wissenschaftlichen Arbeitens folgt. Als Jürgen Habermas im Jahre 1957 „Die chronischen Leiden der Hochschulreform“ veröffentlichte, sprach er von didaktischen Seitenwegen in der Hochschullehre (vgl. Habermas 1981, S. 38). Das Wort Seitenweg soll an dieser Stelle verdeutlichen, dass guter Lehre im akademischen Alltag immer Respekt entgegengebracht wurde, insbesondere seitens der Studierenden, die Karrierepfade aber in viel stärkerem Maße von den erbrachten Forschungsleistungen abhängig gemacht wurden. Im weiteren Sinne gehört dazu auch das Publikationsaufkommen, heute verstärkt darauf basierende Indikatoren, die den Vergleich des Leistungsspektrums konkurrierender Kandidatinnen und Kandidaten ermöglichen sollen. In Berufungsverfahren werden diese zwar seit einigen Jahren in der Regel aufgefordert, auch Ergebnisse ihrer Lehrevaluation vorzulegen, aber von einer ausgewogenen Bewertung von Lehre und Forschung ist der Alltag der Rekrutierung wissenschaftlichen Personals noch etwas entfernt. Die Chance, dass über die vielen neuen Lehr- und Lerninitiativen das Didaktische aus einer Nische mehr in die Mitte gerückt wird, steht mit Blick auf die digitale Lehre durchaus nicht schlecht. Signale, dass es hier vor allem auch um Anerkennung geht, sind an verschiedenen Stellen zu spüren. Als Beispiel sei das im Rahmen des Hochschulforums Digitalisierung initiierte „HFD Certificate“ genannt. ${ }^{1}$ Es beruht auf einer Peer-to-Peer-Evaluation und soll der Entwicklung neuer didaktischer Konzepte Aufmerksamkeit verschaffen. Unter Anwendung des systemtheoretischen Vokabulars könnte daher gesagt werden: Aus einer Minimalinklusion der Vergangenheit wird mittelfristig ein festes Strukturmerkmal (vgl. zu diesen Begriffen Stichweh 2020).

Je facettenreicher das Lehren im Rahmen dieses Prozesses wird, desto stärker werden auch die Grenzen zwischen Lehren und Lernen verwischen. Kollaborative Formen stoßen zahlenmäßig zwar an ihre Grenzen, aber die Strukturen der Vergangenheit - Vortrag, Fragerunde; Vortrag, Fragerunde; ... - lassen den Wissensraum, in dem man sich bewegt, offener wirken als zuvor.

\footnotetext{
${ }^{1}$ Siehe Website des HFDcert: https://hfdcert.de/. Zugegriffen: 17.11.2020.
} 
Dennoch ist es am Ende in der Regel nicht die Gruppe, die prämiert wird, sondern die Leistung einer einzelnen Person. Das gilt für die Seite der Lehrenden ebenso wie für die Seite der Lernenden, auch wenn sie über das Interesse an bestimmten Themen oder Forschungsgebieten zueinanderkommen. „Openness“ ist zu einer verheißungsvollen Formel und zu einer Herausforderung geworden. Der ungehinderte Zugang zu Wissen aller Art ist als Forderung berechtigt, aber die Urheberschaft dürfte nicht immer einem Kollektiv zuzuschreiben sein. Der digitale Humanismus stößt auch dort an seine Grenzen, wo Leistungen individuell zugerechnet werden sollen.

Die Wertschätzung der Didaktik kann im Ergebnis natürlich dazu führen, dass eine Universität oder eine Hochschule aufgrund ihrer guten Lehre insgesamt zertifiziert wird. Dass davon Pionier*innen und Trittbrettfahrer*innen profitieren, lässt sich kaum vermeiden. Eine so gestaltete Evaluation der Lehre ist aber auch deshalb wichtig, weil außerhalb der akademischen Mauern viele andere akademische Plattformen mit durchaus hohen Nutzungszahlen Akzeptanz verbuchen können. Sie werden die akademische Lehre nicht ersetzen, aber aufgrund ihrer Existenz auch dieses System herausfordern. Je unübersichtlicher die Welt der neuen Abschlüsse (Micro-Degrees, Nano-Degrees) sein wird, desto mehr wird auch das Zeugnis und Abschlusswesen der etablierten Institutionen Wertschätzung differenziert abbilden müssen.

\subsection{Methoden und Forschungsdesigns}

Für eine angemessene Einschätzung der Gesamtlage ist es des Weiteren hilfreich, die Verzahnung analoger und digitaler Elemente in der Lehre als einen evolutionären Vorgang zu betrachten. Deshalb soll der Blick auf Methoden- und Forschungsdesigns dafür sensibilisieren, dass nahezu alle wissenschaftlichen Disziplinen kreative Impulse durch neue Beobachtungs- und Analyseinstrumente erhalten haben. Diese neuen Impulse finden Eingang in Lehrinhalte und verändern damit den Gegenstand von Vorlesungen, Seminaren und Übungen. Wenige Beispiele sollen dies verdeutlichen:

- Das Stichwort „Digital Sociology“ steht unter anderem für die Vorstellung, dass viele Spuren des gesellschaftlichen Lebens beobachtet werden können, ohne reaktive Verfahren (zum Beispiel Befragungen) einzusetzen. Ebenso ist die Idee der Netzwerkanalyse, ursprünglich im Kontext der Analyse von Kleingruppenprozessen entstanden, zu einem Synonym für die Analyse sozialer Wechselwirkungen in elektronisch bestimmten Umgebungen geworden. 
- In der Diskussion um die Bedeutung der Künstlichen Intelligenz wird nicht nur die zunehmende Bedeutung von Algorithmen hervorgehoben, sondern die lange Historie dieses Forschungsfelds betont, das unter den heutigen Umständen seine ,zweite Luft“ bekommt.

- In dem weiten Feld der Literaturwissenschaft steht „Digital Humanities“ als Sinnbild einer durchaus nicht konfliktfreien Sicht auf den Kern geisteswissenschaftlicher Textkorpora. Die „maschinelle“ Ausweitung von Vergleichsebenen der Literatur mündet hier in Kontroversen über Sinn und Zweck dieser Optionen.

Da Lehre und Forschung nicht distinkte Säulen, sondern etwas Zusammenhängendes darstellen, hat allein die Veränderung von Erwartungen an die Gestaltung und Dokumentation von Forschungsprozessen den Aufbau neuer digitaler Kompetenzen, zum Beispiel im Forschungsdatenmanagement, aber auch im Publikationswesen, notwendig gemacht. In der Zusammenschau erweisen sich daher viele Hochschulen und Universitäten weitaus digitaler, als sie in der Öffentlichkeit wahrgenommen werden.

\section{$4 \quad$ Fazit}

$\mathrm{Zu}$ Beginn dieses Beitrags wurde auf die große Bedeutung der Kategorie „Wissen“ für die Selbstbeschreibung moderner Gesellschaften hingewiesen. Überblicksbeiträge zur Wissenssoziologie erinnern regelmäßig an die Seinsverbundenheit des Wissens und des Denkens (siehe hierzu beispielsweise die Beiträge in Mannheim 1980). Gemeint ist, dass Wissen qua Existenz eine Gesellschaft nicht in gleicher Weise erfasst. Das Wissen und seine Gesellschaft bedeuten in diesem Zusammenhang die Koexistenz von Nähe und Distanz zu geglaubten und bewiesenen Gewissheiten. Die Wissenschaft kennt aufgrund ihrer Verfasstheit keine lokalen Grenzen. Das Prinzip der Offenheit (siehe oben) ist, im Rahmen der Austauschmöglichkeiten, global. Trotz dieser Offenheit hat sich die Universität als Institution stets zwischen organisatorischer Stabilität und wissenschaftlicher Beweglichkeit weiterentwickelt. Diese Organisationsform des Wissens hat, wie das Wissen selbst, eine soziale Seite. Für ihr Verhältnis zur Digitalisierung folgt daraus, dass die damit verbundenen Herausforderungen nach den Regeln der Institution integriert werden.

Als der Medienphilosoph Claus Pias im Juli 2019 seinen Beitrag „Die Digitalisierung gibt es nicht" veröffentlichte, wird mancher, bevor er überhaupt zu lesen begann, in Erstaunen versetzt worden sein. Der Beitrag selbst aber hatte das 
Ziel, das historische Bewusstsein für dieses Thema wiederzubeleben. An einer Stelle heißt es: „Denn ,Digitalisierung‘ selbst kennt die Zukunft nur als ständig drängendes Update einer sich in ihr stets wiederholenden Gegenwart" (Pias 2019, S. N4). Wer auch immer in Zukunft durch die digitalisierte Hochschullandschaft reist und sich ein Bild von der Lage machen möchte, sollte daher bedenken: Die Digitalisierung alleine organisiert gar nichts. Aber das System ist in Bewegung.

\section{Literatur}

Assheuer, T. (3. Januar 2019). Denken ist keine Sünde. Die Zeit, 2, 42.

Beck, U., \& Beck-Gernsheim, E. (Hrsg.). (1994). Riskante Freiheiten. Individualisierung in modernen Gesellschaften. Frankfurt a. M.: Suhrkamp.

Blumenberg, H. (2001). Licht als Metapher der Wahrheit. In H. Blumenberg (Hrsg.), Ästhetische und metaphorologische Schriften (S. 139-171). Frankfurt a. M.: Suhrkamp.

Habermas, J. (1981). Die chronischen Leiden der Hochschulreform. [Erstveröffentlichung 1957]. In J. Habermas (Hrsg.), Kleine politische Schriften I-V (S. 13-40). Frankfurt a. M.: Suhrkamp.

Kahl, R. (1999). Der Neugierologe. Geo Wissen, 1, S. 106-109.

Kaube, J. (5. Januar 2020). Zurück in die Zukunft. Frankfurter Allgemeine Zeitung, 1, 33.

Knoblauch, H., \& Tuma, R. (2018). Wissen. In J. Kopp \& A. Steinbach (Hrsg.), Grundbegriffe der Soziologie (12. Aufl., S. 517-519). Wiesbaden: Springer VS.

Knoblauch, H. (2008). Wissen. In N. Baur, H. Korte, M. Löw, \& M. Schroer (Hrsg.), Handbuch Soziologie (S. 465-481). Wiesbaden: VS.

Koselleck, R. (1990). Einleitung - Zur anthropologischen und semantischen Struktur der Bildung. In R. Koselleck (Hrsg.), Bildungsbürgertum im 19. Jahrhundert. Teil 2: Bildungsgüter und Bildungswissen (S. 11-46). Stuttgart: Klett-Cotta.

Lyotard, J.-F. (1999). Das postmoderne Wissen. Ein Bericht. [Aus d. Franz., Erstveröffentlichung 1979] (4. Aufl.). Wien: Passagen-Verlag.

Mannheim, K. (1980). Strukturen des Denkens. Frankfurt a. M.: Suhrkamp.

McLuhan, M. (1968). Die magischen Kanäle. Understanding Media. [Aus d. Amerik.]. Düsseldorf, Wien: Econ.

Merton, R. K. (1972). Wissenschaft und demokratische Sozialstruktur. In P. Weingart (Hrsg.), Wissenschaftssoziologie 1. Wissenschaftliche Entwicklung als sozialer Prozess (S. 45-59). Frankfurt a. M.: Suhrkamp.

Pias, C. (31. Juli 2019). Die Digitalisierung gibt es nicht. Frankfurter Allgemeine Zeitung, 175, S. N4.

Rexroth, F. (25. September 2019). Korporative Identität. Frankfurter Allgemeine Zeitung, 223, S. N3.

Stichweh, R. (2020). Unablässige Prozesse. Inklusion, Exklusion und die Differenzierungsdynamik der modernen Gesellschaft. Forschung \& Lehre, 4, 298-300. 
Open Access Dieses Kapitel wird unter der Creative Commons Namensnennung 4.0 International Lizenz (http://creativecommons.org/licenses/by/4.0/deed.de) veröffentlicht, welche die Nutzung, Vervielfältigung, Bearbeitung, Verbreitung und Wiedergabe in jeglichem Medium und Format erlaubt, sofern Sie den/die ursprünglichen Autor(en) und die Quelle ordnungsgemäß nennen, einen Link zur Creative Commons Lizenz beifügen und angeben, ob Änderungen vorgenommen wurden.

Die in diesem Kapitel enthaltenen Bilder und sonstiges Drittmaterial unterliegen ebenfalls der genannten Creative Commons Lizenz, sofern sich aus der Abbildungslegende nichts anderes ergibt. Sofern das betreffende Material nicht unter der genannten Creative Commons Lizenz steht und die betreffende Handlung nicht nach gesetzlichen Vorschriften erlaubt ist, ist für die oben aufgeführten Weiterverwendungen des Materials die Einwilligung des jeweiligen Rechteinhabers einzuholen.

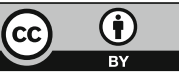

